\title{
公示语英译实践中的文化转向和翻译转向思考
}

\author{
王乔 \\ 西北政法大学 \\ DOI:10.32629/er.v3i4.2614
}

[ 摘 要] 本文从西方翻译研究学派中文化学派理论的界定入手, 以2017年11月20日发布的《公共服务领域英文译写规范》及笔者自身参与公示 语英译实践中搜集的资料为语料,在多元系统学派创始人佐哈尔的翻译理论大背景下,对比文化学派巴斯奈特等人的文化转向理论分析文化、语 言与翻译的关系,思考翻译的意义、价值和目的,旨在思考文化转向的基础上为构建 “翻译转向” 提供思路和建议。

[关键词] 公示语英译; 文化转向; 翻译转向

近年来, 外宣翻译在我国对外文化交流中凸显出十分重要的地位, 公 示语英译作为外宣翻译的一种, 不仅是外籍游客来华能否快速得到所需服 务和信息的重要保障, 同时也是反映我国对外文化建设的一面镜子。我国 首个关于外语在境内如何规范使用的系列国家标准《公共服务领域英文译 写规范》(以下简称《标准》) 由国家质检总局、国家标准委于 2017 年 11 月20日联合发布, 自2017年12月1日起正式实施, 该《标准》规定文化、娱 乐、体育等共 13 个服务领域英文译写的原则、方法和要求, 并提供常用的 上万条规范译文, 但同时对公示语英译实践与研究的影响、翻译研究的文 化转向与翻译实践中的翻译转向提供思路。

\section{1 文化转向的理论流变}

自解构主义运动以来, 语言学派理论中的核心概念 “对等” (Equivalance) 开始遭到严重的质疑。玛丽・斯内尔・霍恩比曾批判, ““对 等, 不再适合作为翻译理论的一个根本概念, “对等” 这个术语, 除了不准 确和界定模糊之外, 它还给人一种错觉, 那就是语言之间是彼此对称的, 而 语言之间除去模糊的对应关系外不存在这种对称。” (Mary Sne11-Hornby, 1988: 22) 翻译研究学派的先驱之一, 多元系统学派的创始 人佐哈尔将翻译文学作为其重要的考察对象之一, 借助俄国形式主义文 艺理论的多层次文学体系说, 深入观察翻译对社会的影响, 从而提出 “多 元系统” 理论。20世纪60、70年代, 翻译文化转向已经初现于众多语言 学理论中。

1972年, 翻译研究学派代表人物之一詹姆斯 - 霍姆斯提出以翻译研究 (translation studies) 这个术语作为学科名称, 此举不但为翻译学科正 名, 也让翻译学科研究更多元, 更促进翻译研究的转向。1990年文化学派重 要代表巴斯奈特与勒弗维尔合编一本论文集《翻译、历史与文化》 (Translation, History, Culture), 从宏观角度出发, 以政治、文学、文化、 意识形态等多角度切入, 正式提出 “将翻译视为文化与政治的活动的的转 向” 这一口号, 霍恩比在被上述论文集收录的论文中将其称为 “文化转向” (Cu1tural Turn), 巴斯奈特与勒弗维尔采纳了这一术语 (杰里米 - 芒 笛, 2014: 181), 翻译研究学派开始进入 “翻译的文化转向” 阶段, 文化转

但又隐去了作者的主观迹象。由于作者没有提供更多实际证据, 很有可 能只是作者猜测, 但却以中国的立场表述, 故意给读者造成真实、客观的 感觉。

\section{3 结语}

新闻英语由于特有的文本特征, 存在大量语法隐喻, 这些隐喻在新闻 语篇的客观和简洁表达、达成作者写作意图、以及语篇衔接方面发挥了重 要作用。对语法隐喻的解读还有利于更深入理解作者的深层次含义, 也是 理解语篇的重要途径。
向研究在 20 世纪 80 年代的西方翻译理论界呈现出蔚为壮观之势。中国翻译 界在晚于西方大致十年后也实现了翻译研究的文化转向。国内翻译理论学 者张思永通过对翻译理论研究的文化转向之趋势进行梳理和预测, 将翻译 领域的文化研究大体上分为翻译实践的文化研究、翻译活动的文化研究及 翻译理论的文化研究三类。

\section{2 文化走出去大背景下的文化、语言与翻译关系的思考}

2.1文化、语言与翻译的关系

首先, 文化和语言体现在两个方面:一是语言是文化的载体, 二是文化 是语言的结晶。“语言是翻译中的核心问题, 言语是包括文化信息在内的一 切信息的载体, 所以文化研究也必须通过对文本的文化解读获得, 而不是 通过外在性研究获取。” (吕俊、侯向群, 2010) 其次, 语言作为翻译的工具, 翻译是语言的阐释。纽马克指出翻译理论的核心是翻译问题。“翻译研究 的文化转向” 提出, 是为了让人们认识到从语言角度对语言进行详细阐释, 并探讨翻译的局限性, 拓宽翻译研究的范围。因此, 在翻译和语言二者的关 系中, 以语言为工具, 以翻译的哲学意义为导向, 将翻译研究从 “散落在其 他较为古老的学科之中” (杰里米 - 芒笛, 2014：17) 变成散落在其他新兴 学科之中, 才能真正达到语言与翻译二者的共生。最后, 文化是翻译的前提, 翻译是对文化的传承, 翻译绝不是一个纯语言的行为, 而是深深植根语言 所处的文化之中。勒弗维尔认为, “翻译就是文化内与文化间的交流”, “翻译等值就是原语与译语在文化功能上的等值” (Bassnett \& Lefevere, 1992：8）。正因如此, 翻译应以文化作为翻译单位, 为文化交流 也包括文化之间相互交流。

2.2 “文化转向” 向 “翻译转向” 迈进中的翻译缺失

“文化转向” 强调文化在翻译中的地位, 将对翻译的内部研究转向对 翻译的外部研究。文化研究的深入使学者们注意到语言问题、翻译问题, 并把翻译作为文化研究的资源, 形成所谓的文化研究的翻译转向, 从而从 外部促进了翻译研究的文化转向 (谢天振, 2006: 5)。笔者认为是文学研究 的兴起和文化学派的文化意识的觉醒共同刺激了翻译研究的文化转向。因 为 “翻译转向” 让学者们开始把翻译放在政治、文化的大背景下研究, 使

\section{[参考文献]}

[1]Ha71iday M.A.K.Language as social semiotic: The social interpretation of language and meaning[M].London:Arnold,1978.

[2]张德禄,雷茜.语法隐喻研究在中国[]].外语教学,2013,34(3):1-6.

[3]宁志敏。语法隐喻在报刊英语中的语篇作用 [J]. 英语研 究,2005,(1):19-23.

[4]周大军. 英语的情态隐喻及其语篇解释力[]]. 四川外语学院学报, 四 川外语学院学报,2003,(06): 100-104. 
多元的学科得以交叉研究, 但因文化研究的 “翻译转向” 焦点仍大多集中 在文化对翻译活动的控制, 所以翻译研究中的文化转向和文化研究中的翻 译转向并没有达到平等。

\section{3 从公示语英译视角看文化走出去的两大桎梏}

笔者在参与公示语英译实践中和对《标准》的学习中发现, 公示语英 译所体现出的问题在于语言规范问题多, 例如, 违反合法性原则、违反准确 性原则、违反规范性原则、违反通俗性原则, 以及警示和提示信息的译文 未兼顾文明性原则, 公共应急指示标识不够完善。而在实践过程中, 即使如 今的《标准》为公示语英译提出规范性意见, 但对于翻译学习者和翻译研 究者而言, 这并非代表公示语英译可以就此停止研究。因此, 公示语英译问 题多且没有作用于翻译学习者和翻译研究者成为文化走出去的两大桎梏。

\section{3 将文化转向落实到翻译转向的策略提出}

3. 1 推动翻译转向, 重拾文化经典

德贾斯维尼 - 尼南贾纳指出: “人类学家为自己制定的任务就是文化 之间的翻译, 即把一种文化翻译成另一种文化能够理解的术语。” (张京 媛, 1999) 为了让我国翻译历史任务在翻译转向的过程中既不丧失主流意 识, 笔者认为应当重拾我国的传统文化、鼓励经典国学作品阅读、重视文 化典籍翻译, 并对经典国学作品翻译的不同版本进行赏析研究, 把翻译看 作是一种文化发展的策略来研究。

3.2把握翻译转向时机, 推动国内翻译流派的建立

原本狭义的翻译研究忽视了翻译活动中蕴含的权力关系和话语研究。 在我国处于亟需发生翻译转向时, 应该在考虑翻译文本的历史问题或历史 具有有效性原则的基础上, 把握时机, 梳理和归纳积累多年来我国学者的 译学研究, 建立起我国的翻译流派更加符合我国传统译学与译论建设的实 际情况。

3. 3 审视理论实践的关系, 落实翻译人才培养

笔者认为是语言规范问题多是导致理论实践不平衡的原因, 而理论实 践不平衡又反之让语言规范问题多的问题得不到解决。原因有三: 第一, 翻译实践在文化传播的影响下加速, 翻译理论的研究来不及为翻译实践提 供有效的理论指导; 第二, 许多翻译研究者过分关注对文化的研究而忽视 对实际翻译行为和翻译活动本身的研究, 造成翻译的理论体系与翻译实践 脱节, 也就是说, 我们一味的做研究, 忽视对翻译实践的纠正和思考; 第三, 翻译实践者的输出与翻译者自身的翻译理论的输入不成正比, 这也许是因 为翻译教学中, 教师对翻译理论与实践研究的分配和把握有所不同, 亦或 是 “重理论轻实践”、“没有实践何来理论”, “没有理论如何实践” 的悖 论形成的矛盾。基于以上三个原因的分析, 笔者针对文化转向的背景, 从文
化走出去与语言规范问题多及理论实践不平衡相矛盾的角度提出三点意 见:（1）重新探索文化翻译和翻译文化的关系; (2) 公示语英译或外宣翻译 人才应担负起 “文化大使” 的角色; (3) 翻译学习者应当提升文化敏感度, 广泛阅读, 学习和鉴赏国学, 培养文化责任感。针对翻译转向倾向, 从文化 走出去与翻译人才培养的角度提出两点意见: (1) 进一步思考MTI课程设置, 尤其注重典籍翻译的涉及和译者语言功底的培养。(2) 就翻译课程教授者 而言, 教师应当重新思考教学准备和安排, 多引导学生有效思考和阅读, 激 发学生的探索和求知欲, 将身边的翻译素材引进课堂。

\section{4 结束语}

综上所述, 无论是外宣翻译还是公示语英译都值得我们从文化转向理 论角度出发深入思考, 国内翻译理论研究的深度和广度已处于文化转向的 进程中, 笔者在结合文化转向理论, 分析《标准》与自身参与的公示语英译 语料, 指出我国文化走出去的两大桎梏, 针对语言、文化和翻译的关系, 阐 释出将 “文化转向” 向 “翻译转向” 拓展, 并结合其发展所带来的思考, 表明在文化转向的大背景下, 国内翻译研究应关注非精英文化的文化研究, 以此可表明未来文化研究的发展道路之一就是通过阐释性的文化翻译使 文化变得越来越多元化的道路, 从翻译学习者和翻译学研究者的角度提出 “翻译转向” 的优化策略, 进而推动我国翻译学研究和翻译流派的建立。

\section{[参考文献]}

[1]Andre Lefevere. Translation, Rewriting, and the Manipulation of Literature[M]. Routledge,1992.

[2]Mary Sne11-Hornby.Translation Studies: An Integrated Approach[M] Amsterdam/Philadelphia:John Benjamin Publishing Company,1988.

[3]国家质检总局、国家标准委.公共服务领域英文译写规范 [S].2017-11-20

[4]吕俊, 侯向群.范式转换抑或视角转变一一与谢天振教授商榷 [J].中 国翻译,2010(1):41-45.

[5]杰里米- 芒笛著, 李德风等译. 翻译学导论: 理论与实践(第三版) [M]. 北京:外语教学与研究出版社,2014.

[6]谢天振。翻译研究的 “文化转向”之后——翻译研究文化转向的 比较文学意义[J].中国比较文学,2006(3):1-14.

[7]张京媛. 后殖民理论与文化批评[M]. 北京: 北京大学出版社, 1999 .

\section{作者简介:}

王乔(1994--),女,汉族,宁夏固原人,硕士在读,西北政法大学翻译 硕士专业, 研究方向：英语笔译。 